[8] S. K. Kaul, A characterization of Čech homology theory, Colloq. Math. 21 (1970), pp. 229-237. [9] Y. Kodama, On the shape of decomposition spaces, J. Math. Soc. Japan 26 (4) (1974).

[10] - On A spaces and fundamental dimension in the sense of Borsuk, Fund. Math. 89 (1975) pp. 13-22

11] - On embeddings of spaces into ANR and shapes, to appear.

[12] G. Kozlowski and J. Segal, On the shape of 0-dimensional paracompacta, Fund. Math. 83 (1974), pp. 151-154.

[13] S. Mardešić, Shapes for topological spaces, Gen. Top. and Appl. 3 (1973), pp. 265-282 [14] S. Scion. Sér. Sci. Math. Astronom. Phys. 21 (1973), pp. 1137-1142.

[15] E. Michael, Another note on paracompact spaces, Proc. Amer. Math. Soc. 8 (1957), pp. $822-828$.

TOKYO UNIVERSITY OF EDUCATION

\section{Some uniformization results}

by

\section{H. Sarbadhikari (Calcutta)}

Abstract. Some results on the uniformization of Borel sets are proved in this paper.

1. Introduction. Let $X, Y$ be Polish spaces and $B \subseteq X \times Y$. We say $C$ uniformizes $B$ if $C \subseteq B$ and for all $x \in \operatorname{pr}_{X} B, C \cap B^{x}$ is a singleton where $B^{x}$ $=\{y:(x, y) \in B\}$ and $\operatorname{pr}_{X} B$ is the projection of $B$ to $X$. In general, a Borel set $B$ does not have a Borel uniformization ([2], [6]). However, in some cases, such a uniformization exists, for example, if $B^{x}$ is $\sigma$-compact for each $x$ [1] or if $\mu\left(B^{x}\right)>0$ for each $x$ where $\mu$ is a probability measure on the Borel $\sigma$-algebra of $Y$ [3].

The chief aim of this paper is to prove the following:

THEOREM 1. Let $X, Y$ be Polish spaces and $B \subseteq X \times Y$ be Borel. $B$ has a Borel uniformization if any one of the following is true:

(1) for all $x \in \operatorname{pr}_{x} B, B^{x}$ contains an isolated point

(2) for all $x \in \mathrm{pr}_{X} B, B^{x}$ contains a point which is not its point of condensation,

(3) for all $x \in \mathrm{pr}_{X} B, B^{x}$ is not meager.

The paper is organized in the following way. Section 2 is devoted to preliminaries. In Section 3, a proof of Theorem. 1 is given. In Section 4, a related result is proved.

2. Preliminaries. A set is called meager if it is a countable union of nowhere dense sets. A comeager set is one whose complement is meager. Let $X, Y$ be Polish spaces, $B \subseteq X \times Y$ and $U \subseteq Y$. Following Vaught, we put $B_{U}^{*}=\left\{x: B^{x} \cap U\right.$ is comeager in $U\}$. It is known that if $B$ is Borel and $U$ open, then $B_{U}^{*}$ is Borel [7]. For any set $A$, let $\delta(A)$ denote the diameter of $A$.

If $f$ is a function, put $Z_{f^{\prime}}=\left\{y: f^{-1}(y)\right.$ is a singleton $\}, I_{f}=\left\{y: f^{-1}(y)\right.$ contains an isolated point $\}, D_{f}=\left\{y: f^{-1}(y)\right.$ is countable and non-empty $\}, C_{f}=\left\{y: f^{-1}(y)\right.$ contains a point which is not its condensation point\}. It is known that if $f$ is a Bore measurable function defined on a Borel subset of a Polish space into a separable metric space, then $Z_{f}, I_{f}, D_{f}, C_{f}$ are coanalytic [4].

\section{Proof of main theorem.}

Proof of (1). Let $\left\{V_{n}\right\}$ be a countable open base for $Y$. For any $n$, define $f_{n}$ on $B \cap\left(X \times V_{n}\right)$ by $f_{n}(x, y)=x$. Let $Z_{n}=\left\{x: B^{x} \cap V_{n}\right.$ is a singleton $\}$. Then $Z_{n}=Z_{f_{n}}$ 
is coanalytic and $\operatorname{pr}_{X} B=\bigcup_{n} Z_{n}$ is Borel. Let $B_{n} \subseteq Z_{n}$ be disjoint Borel sets such that $\operatorname{pr}_{X} B=\bigcup_{n} B_{n}$. Let $C=\bigcup_{n}\left(\left(B_{n} \times V_{n}\right) \cap B\right)$. Then $C$ is a Borel uniformization of $B$.

Proof of (2). Let $\left\{V_{n}\right\}$ and $\left\{f_{n}\right\}$ be defined as above and, for any $n$, let $Z_{n}$ $=\left\{x: B^{x} \cap V_{n}\right.$ is countable and non-empty $\}$. Then $Z_{n}=D_{f_{n}}$ is coanalytic and $\operatorname{pr}_{X} B=\bigcup_{n} Z_{n}$ is Borel. Let $B_{n}$ be as before. Let $D=\bigcup_{n}\left(\left(B_{n} \times V_{n}\right) \cap B\right)$. Then $D \subseteq B$ is " a Borel set such that for $x \in \operatorname{pr}_{X} B, D^{x}$ is non-empty and countable. $D$ can therefore be uniformized by a Borel set $C$, which also uniformizes $B$.

Remark. Proofs of (1) and (2) illustrate the use of the reduction principle in proving selection theorems. For a detailed exposition see [5].

Pro of of (3). This follows from:

THEOREM 2. If $X, Y$ are Polish spaces and $B \subseteq X \times Y$ is a Borel set such that for all $x \in \operatorname{pr}_{X} B, B^{x}$ is comeager, then $B$ has a Borel uniformization.

Assuming Theorem 2, we prove (3) as follows: Suppose $B \subseteq X \times Y$ is a Borel set such that for all $x \in \operatorname{pr}_{X} B, B^{x}$ is not meager. Let $\left\{V_{n}\right\}$ be a countable open base for $Y$. Put $D_{n}=B_{V_{n}}^{*}-\bigcup B_{V_{m}}^{*}$ for all $n$. By Theorem 2, the Borel subset $B \cap\left(D_{n} \times V_{n}\right)$ of $X \times V_{n}$ can be uniformized by a Borel set $C_{n}$. As $\operatorname{pr}_{X} B=\bigcup_{n} B_{V_{n}}^{*}=\bigcup_{n} D_{n}$ [7],

$C=\cup C_{n}$ uniformizes $B$. This concludes the proof of Theorem 1 .

(1) and (2) yield the following:

Corollary. Let $X$ be absolutely Borel and $Y$ a separable metric space. Let $f: X \rightarrow Y$ be Borel measurable. If $Y=I_{f}$ or $C_{f}$, then $f$ admits a Borel selector, i.e., there is a Borel subset $B$ of $X$ such that $f$ restricted to $B$ is one-to-one and $f(B)$ $=f(X)=Y$.

Remark. (3) is a category analogue of the result of Blackwell and Ryll-Nardzewski [3] referred to in the introduction.

Proof of Theorem 2.

LeMma. Let $X, Y$ be Polish spaces and $B \subseteq X \times Y$ be Borel. Given any open subset $U$ of $Y$, there is a sequence $\left\{Z_{k}\right\}$ of subsets of $X \times Y$ satisfying the following:

(a) Each $Z_{k}$ is a Borel subset of $X \times U$.

(b) $\cap Z_{k} \subseteq B$.

(c) Given any non-empty open $W \subseteq U$, any $k$ and any $8>0$, there is a Borel set $F \subseteq Z_{k} \cap(X \times W)$ such that for all $x, F^{x}$ is closed, $\delta\left(F^{*}\right)<\varepsilon$ and if $x \in B_{U}^{*}$, then $F^{*}$ is not meager.

Pro of. Let $M=\{B \subseteq X \times Y: B$ is Borel and satisfies the above $\}$. We show that $M$ contains all Borel sets.

Step 1. $M$ contains closed sets.

Let $\left\{W_{m}\right\}$ and $\left\{V_{n}\right\}$ be countable open bases for $X$ and $Y$ respectively. Let $B \subseteq X \times Y$ be closed. Then there are open sets $U_{k} \subseteq X \times Y, k=1,2, \ldots$ such that
$B=\bigcap_{k} U_{k}$. Let $U \subseteq Y$ be open. Put $Z_{k}=U_{k} \cap(X \times U)$ for all $k$. Clearly, $\left\{Z_{k}\right\}$ satisfies (a) and (b). To see that it also satisfies (c), fix any $k$, any $\varepsilon>0$ and any nonempty open subset $W$ of $U$. We shall construct $F$ so that (c) is satisfied.

$Z_{k} \cap(X \times W)=U_{k} \cap(X \times W)$ is open and hence a countable union of sets of the form $W_{m} \times V_{n}$. Let $L=\left\{m: W_{m}\right.$ appears in this union $\}$. Corresponding to each $m \in L$, choose exactly one $n$ such that $W_{m} \times V_{n}$ appears in this union and let $V_{m} \neq \varnothing$ satisfy $V_{m} \subseteq \widetilde{V}_{m} \subseteq V_{n}$ and $\delta\left(V_{m}\right)<\varepsilon$. Let

$$
F=\bigcup_{m \in L}\left(W_{m}-\bigcup_{\substack{n<m \\ n \in L}} W_{n}\right) \times \bar{V}_{m} .
$$

Step 2. $M$ is closed under countable intersections.

Let $B_{n} \in M$ for $n=1,2, \ldots$ and let $U \subseteq Y$ be open. For each $n$, let the sequence of sets $Z_{n k}, k=1,2, \ldots$ satisfy (a), (b), (c) if $B$ is replaced by $B_{n}$ and $Z_{k}$ by $Z_{n k}$ Rearrange the double sequence $\left\{Z_{n k}\right\}$ in the form of a simple sequence $\left\{Z_{k}\right\}$. Then $\left\{Z_{k}\right\}$ satisfies (a), (b), (c) if $B=\bigcap_{n} B_{n}$.

Step 3. $M$ is closed under countable unions.

Let $B_{n} \in M$ for $n=1,2, \ldots$ and let $U \subseteq Y$ be open. We construct a sequence $\left\{Z_{k}\right\}$ satisfying (a), (b), (c) if $B=\cup B_{n}$.

Lat $\left\{V_{m}\right\}$ be a countable open base for $U$. For each $m, n$, let $Z_{n m k}, k=1,2, \ldots$ satisfy (a), (b), (c) with $B$ replaced by $B_{n}, U$ by $V_{m}$ and $Z_{k}$ by $Z_{n m k}$. For all $n, m, k$ put

$$
\begin{array}{cc}
D_{m m}=B_{n V_{m}}^{*}-\bigcup_{j<n} B_{j V_{m}}^{*}, & E_{m}=\bigcup_{n} D_{n m}=\bigcup_{n} B_{n V_{m}}^{*}, \\
Z_{m k}=\bigcup_{n}\left(Z_{m m k} \cap\left(D_{n m} \times Y\right)\right), & Z_{k}=\bigcup_{m}\left(Z_{m k}-\bigcup_{i<m}\left(E_{i} \times V_{i}\right)\right) .
\end{array}
$$

Clearly, $Z_{k}$ is a Borel subset of $X \times U$ for each $k$.

$$
\bigcap_{k} Z_{k}=\bigcap_{k} \bigcup_{m}\left(Z_{m k}-\bigcup_{i<m}\left(E_{i} \times V_{i}\right)\right)=\bigcup_{m} \bigcap_{k}\left(Z_{m k}-\bigcup_{i<m}\left(E_{i} \times V_{i}\right)\right)
$$

since $Z_{m k} \subseteq E_{m} \times V_{m}$ for all $k$ and $m$.

For any $m$,

$$
\begin{aligned}
\bigcap_{k}\left(Z_{m k}-\bigcup_{i<m}\left(E_{i} \times V_{i}\right)\right) & =\bigcap_{k} \bigcup_{n}\left(Z_{n m k} \cap\left(D_{n m n} \times Y\right)-\bigcup_{i<m}\left(E_{i} \times V_{i}\right)\right) \\
& =\bigcup_{n} \bigcap_{k}\left(Z_{i m k} \cap\left(D_{m m} \times Y\right)-\bigcup_{i<m}\left(E_{i} \times V_{i}\right)\right)
\end{aligned}
$$

since $D_{m m}, n=1,2, \ldots$ is a disjoint sequence.

Thus

$$
\bigcap_{k} Z_{k} \subseteq \bigcup_{m} \bigcup_{n} \bigcap_{k} Z_{u m k} \subseteq \bigcup_{n} B_{n}
$$

Fix any $k$, any $\varepsilon>0$ and any non-empty open subset $W$ of $U$. For all $m, n$, put

$$
H_{m}=E_{m}-\bigcup_{\substack{i<m \\ W \cap W_{i} \neq \varnothing}} E_{i} \text { and } \quad G_{n m}=H_{m} \cap D_{n m} .
$$


For all $m$ such that $W \cap V_{m} \neq \varnothing$ and all $n$, choose a Borel set $F_{n m} \subseteq Z_{n m k} \cap$ $\cap\left(X \times\left(W \cap V_{m}\right)\right)$ such that for all $x, F_{n m}^{x}$ is closed, $\delta\left(F_{n m}^{x}\right)<\varepsilon$ and if $x \in B_{n V_{m}}^{*}$, then $F_{m m}^{x}$ is not meager. Let

$$
F=\bigcup_{n} \bigcup_{\substack{m \\ W \cap V_{m} \neq \varnothing}}\left(\left(G_{n m} \times Y\right) \cap F_{n m}\right) .
$$

$F$ is clearly a Borel subset of $X \times W$. To see that $F \subseteq Z_{k}$, let $(x, y) \in F$. There exist unique $m$ and $n$ such that $W \cap V_{m} \neq \varnothing$ and $(x, y) \in\left(G_{n m} \times Y\right) \cap F_{n m}$. As $F_{m m} \subseteq Z_{n m k}$ and $G_{m m} \subseteq D_{n m},(x, y) \in Z_{n m k} \cap\left(D_{n m} \times Y\right) \subseteq Z_{m k}$. Let $i<m$. If $W \cap V_{i} \neq \varnothing, x \notin E_{i}$ and if $W \cap V_{i}=\varnothing, y \notin V_{i}$ since $(x, y) \in F_{n m} \subseteq X \times W$ implies $y \in W$. Thus $(x, y)$ $\notin \cup\left(E_{i} \times V_{i}\right)$. Hence $(x, y) \in Z_{k}$.

Clearly $F^{x}$ is closed and $\delta\left(F^{x}\right)<\varepsilon$ for all $x$. Let $\left(\bigcup B_{n}\right)^{x} \cap U$ be comeager in $U$. We show that there is some $m, n$ such that $W \cap V_{m}^{n} \neq \varnothing$ and $x \in G_{n m}$. Thien $F^{x}$ $=F_{n m}^{x}$ and $x \in B_{n V_{m}}^{*}$. Hence $F^{x}$ is not meager.

It is enough to show that there is an $\dot{m}$ such that $W \cap V_{m} \neq \varnothing$ and $x \in E_{m}$. Clearly, $\left(\cup B_{n}\right)^{x} \cap U$ is comeager in $U$ implies $\left(\bigcup B_{n}\right)^{x} \cap W$ is comeager in $W$ so that there is some $n$ for which $B_{n}^{x} \cap W$ is not meager. Hence there is a $V_{m} \subseteq W^{r}$ such that $x \in B_{n V_{m}}^{*} \subseteq E_{m}$.

Proof of the theorem. Let $\left\{V_{n}\right\}$ be a countable base for $Y$. Let $\left\{Z_{k}\right\}$ satisfy (a), (b), (c) of the lemma if $U$ is replaced by $Y$. Let $C_{1} \subseteq Z_{1}$ be a Borel set such that for all $x, C_{1}^{x}$ is closed, $\delta\left(C_{1}^{x}\right)<1$ and for $x \in \operatorname{pr}_{X} B, C_{1}^{x}$ is not meager.

For all $n$, put $H_{1 n}=C_{1 V_{n}}^{*}-\bigcup_{m<n} C_{1 V_{m}}^{*}$ and choose a Borel set $F_{2 n} \subseteq Z_{2} \cap$ $\cap\left(X \times V_{n}\right)$ such that for all $x, F_{2 n}^{x}$ is closed. $\delta\left(F_{2 n}^{x}\right)<\frac{1}{2}$ and $x \in \operatorname{pr}_{X} B$ implies $F_{2 n}^{x}$ is not meager. Let $C_{2}=\bigcup_{n}\left(\left(H_{1 n} \times Y\right) \cap F_{2 n}\right)$. Then $C_{2}$ is a Borel subset of $Z_{2}$. Also $C_{2} \subseteq C_{1}$, for if $x \in H_{1 n}, x \in C_{1 V_{n}}^{*}$ and hence $V_{n} \subseteq C_{1}^{x}$ as $C_{1}^{x}$ is closed; thus $\left(H_{1 n} \times Y\right) \cap$ $\cap F_{2 n} \subseteq H_{1 n} \times V_{n} \subseteq C_{1}$ for all $n$. Clearly, for all $x, C_{2}^{x}$ is closed and $\delta\left(C_{2}^{x}\right)<\frac{1}{2}$. Let $x \in \operatorname{pr}_{X} B$. Then $C_{1}^{x}$ is not meager and therefore $x \in \bigcup_{n} C_{1 V_{n}}^{*}=\bigcup_{n} H_{1 n}$. Hence $C_{2}^{x}$ $=F_{2 n}^{x}$ for some $n$ and therefore is non-meager. By induction, we define a descending sequence $\left\{C_{k}\right\}$ of Borel sets such that for all $k, C_{k} \subseteq Z_{k}$ and for all $x, C_{k}^{x}$ is closed, $\delta\left(C_{k}^{x}\right)<1 / k$ and $x \in \operatorname{pr}_{X} B$ implies $C_{k}^{x}$ is non-meager. $C=\bigcap C_{k}$ uniformizes $B$.

\section{A related result.}

THEOREM 3. Let $X, Y$ be Polish spaces and $B \subseteq X \times Y$ be a Borel set such that for all $x \in \operatorname{pr}_{X} B, B^{x}$ is comeager. Then there exist a sequence $\left\{Z_{k}\right\}$ of Borel sets in $X \times Y$ such that $\bigcap_{k} Z_{k} \subseteq B$ and for all $k$ and $x, Z_{k}^{x}$ is open in $Y$ and $x \in \mathrm{pr}_{X} B$ implies $Z_{k}^{x}$ is denise (and hence comeager) in $Y$.

Remark. Theorem 2 follows from Theorem 3 and the reduction principle.

Theorem 3 is a particular case of:
Theorem 4. Let $X, Y$ be Polish spaces and $B \subseteq X \times Y$ be a Borel set. Given any open $U \subseteq Y$, there is a sequence $\left\{Z_{k}\right\}$ of sets such that

(a) $Z_{k}$ is a Borel subset of $X \times U$ for each $k$

(b) $\cap Z_{k} \subseteq B$.

(c) For all $k$ and $x, Z_{k}^{x}$ is an open subset of $Y$ and if $x \in B_{v}^{*}$, then $Z_{k}^{x}$ is comeager in $U$.

Proof. Let $M=\{B \subseteq X \times Y: B$ is Borel and satisfies the above $\}$. We show that $M$ contains all Borel subsets of $X \times Y$.

Step 1 . Clearly $M$ contains all $G_{\delta}$ sets and hence all closed sets.

Step 2. It is easy to see that $M$ is closed under countable intersections.

Step 3. $M$ is closed under countable unions.

Let $B_{n} \in M$ for $n=1,2, \ldots$ and let $U \subseteq Y$ be open. Let $\left\{V_{m}\right\}$ be a countable open base for $U$. For any fixed $m, n$ let $Z_{n m k}, k=1,2, \ldots$ satisfy (a), (b), (c) if $B$ is replaced by $B_{n}, U$ by $V_{m}$ and $Z_{k}$ by $Z_{m m k}$. For all $m$ and $k$, define $E_{m}$ and $Z_{m k}$ as in the lemma of Section 3 and let $Z_{k}=\bigcup_{m}\left(Z_{m k}-\bigcup_{i<m}\left(E_{i} \times \bar{V}_{i}\right)\right)$. It is easy to see that $\left\{Z_{k}\right\}$ satisfies (a) and (b) if $B=\bigcup_{n} B_{n}$ and that for all $k$ and $x, Z_{k}^{x}$ is open. Let $x$ $\in\left(\cup B_{n}\right)_{U}^{*}$. To show that $Z_{k}^{x}$ is comeager in $U$, it is enough to show that it is dense in $U^{n}$. We prove this in two steps.

Step 1. $V_{x}=\bigcup_{m} V_{m}$ is dense in $U$.

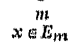

Step 2. $\overline{Z_{k}^{x}} \supseteq V_{x}$.

Proof of Step 1. As $x \in\left(\bigcup_{n} B_{n}\right)_{U}^{*}, x \in\left(\bigcup_{n} B_{n}\right)_{V_{m}}^{*}$ for all $m$. Thus given $m$, there is some $n$ such that $B_{n}^{x} \cap V_{m}{ }^{n}$ is not meager and hence there is some $V_{s} \subseteq V_{m}$ such that $x \in B_{n V_{s}}^{*} \subseteq E_{s}$. Now, $V_{s} \subseteq V_{x} \cap V_{m}$ so that $V_{x} \cap V_{m} \neq \varnothing$.

Proof of Step 2 .

$$
Z_{k}^{x}=\bigcup_{m}\left(Z_{m k}^{x}-\bigcup_{\substack{i<m \\ x \in E_{i}}} \nabla_{i}\right)=\bigcup_{\substack{m \\ x \in E_{m}}}\left(Z_{m k}^{x}-\bigcup_{\substack{i<m \\ x \in E_{i}}} \bar{V}_{i}\right)
$$

If $x \in E_{m}$, there is an $n$ for which $x \in D_{m m}$ so that $Z_{m k}^{x}=Z_{m m k}^{x}$. Hence $\overline{Z_{m k}^{x}} \supseteq \overline{V_{m}}$ and therefore $Z_{m k}^{x}=\widetilde{V}_{m}$. Hence

Thus

$$
\left(Z_{m k}^{x}-\bigcup_{\substack{i<m \\ x \in E_{t}}} \nabla_{i}\right) \geq \nabla_{m}-\bigcup_{\substack{i<m \\ x \in E_{t}}} \nabla_{i} .
$$

$$
\overline{Z_{k}^{x}} \supseteq \bigcup_{\substack{m \\ x \in E_{m}}} \bar{V}_{m} \supseteq V_{x} .
$$

5. Acknowledgment. I am grateful to Dr. Ashok Maitra for his many suggestions and to Dr. B. V. Rao for various discussions. 


\section{References}

[1] V. Ja. Arsenin and A. A. Ljapunov, Theory of A-sets, Uspehi Mat. Nauk 5 (39), (1950), pp. 45-108 (in Russian).

[2] D. B lackwell, A Borel set not containing a graph, Ann. Math. Statist. 39 (1968), pp. 1345-1347.

[3] - and C. Ryll-Nardzewski, Non existence of everywhere proper conditional distributions, Ann. Math. Statist. 34 (1963), pp. 223-225.

[4] K. Kuratowski, Topology, Vol. 1, New York-London-Warszawa 1966.

[5] A. Maitra and B. V. Rao, Selection theorems and the reduction principle, Trans. Amer. Math Soc. 202 (1975), pp. 57-66.

[6] P. Novikoff, Sur les fonctions implicites mesurables B, Fund. Math. 17 (1931), pp. 8-25.

[7] R. Vaught, Invariant sets in topology and logic, Fund. Math. 82 (1974), pp. $269-294$.

INDIAN STATISTICAL INSTITUTE, Calcutta

Accepté par la Rédaction le 25. 8. 1975

\section{On the classification of locally compact separable metric spaces}

by

Gary M. Huckabay * (Lawton, Okla.)

Abstract. Let $M$ be a locally compact separable metric space. Let $P, C$, and $N$ be the space of irrationals, the Cantor set, and the positive integers, respectively. For any space $X$ let $H(X)$ be the homeomorphism group. The existence of standard maps of $P$ onto $M$ and $C \times N$ onto $M$ is established. This provides a classification of the maps of $P$ onto $M$ and $C \times N$ onto $M$ is established, This provides a classification of the locally compact separable metric spaces by
considering certain subgroups of $H(P)$ and $H(C \times N)$.

1. Introduction. A result due to A. R. Vobach [3] completely classifies the compact metric spaces in terms of certain subgroups of the Cantor set. In view of the techniques used by Vobach, it is reasonable to wonder if such results are possible for other classes of spaces with the Cantor set replaced by some other universal space. In this note, we show that this is the case for locally compact separable metric spaces and the irrationals. The author is indebted to Professor Vobach for several valuable suggestions.

2. Preliminaries. Let $N$ be the positive integers with the discrete topology, $C$ any Cantor set, $C^{\prime}$ any Cantor set with a single point removed, and $P$ the space of irrationals on the real line with the subspace topology. For any space $X$, let $H(X)$ denote the full homeomorphism group. Finally, map and $\simeq$ will mean function and homeomorphic, respectively.

LemaA 2.1. If $A_{i}=N$ for each $i$, then $I\left\{A_{i}: i \in N\right\}, C \times P$ and $P$ are homeomorphic.

Proof. To see that $I\left\{A_{i}: i \in N\right\}$ and $P$ are homeomorphic we refer the reader to [2], p. 25, Example 2.

Let $B_{l}=\{0,2\}$. Then $\Pi\left\{B_{l}: i \in N\right\} \simeq C$ and $B_{i} \times N \simeq N$. Therefore

$C \times P \simeq \Pi\left\{B_{l}: i \in N\right\} \times \Pi\left\{A_{l}: i \in N\right\} \simeq \Pi\left\{B_{l} \times A_{l}: i \in N\right\} \simeq \Pi\left\{A_{l}: i \in N\right\} \simeq P$.

LeMma 2.2. Let $X, Y$ and $Z$ be spaces $F: X \rightarrow Y$ an identification, and $G: X \rightarrow Z$ continuous. If $G F^{-1}$ is single-valued, then $G F^{-1}$ is continuous.

* This material will appear in the author's doctoral dissertation, under the direction of Paul F. Duvall, Ir, at Oklahoma State University.

5-Fundamenta Mathematicae XCVIr 